d'habileté du trayeur, car les résultats détaillés du contrôle, traite par traite, ont montré une irrégularité anormale. Quoi qu'il en soit, nous croyons devoir insister sur ce fait que l'accroissement du taux de lipoïdes dans le sang a coïncidé au cours de nos essais avec l'augmentation de la richesse butyreuse du lait.

(A suivre.)

\title{
L'INFLUENCE DES RAYONS ULTRA-VIOLETS SUR LA SÉCRÉ TION LACTÉE CHEZ LA FEMME
}

\author{
par E. LESNÉ et G. DREYFUS-SÉE.
}

Le mécanisme intime de la sécrétion lactée demeure encore mystérieux par nombre de points. En partieulier, les facteurs déterminants quantitatifs et qualitatifs restent inconnus, et par suite la thérapeutique dirigée contre les hypo et les agalacties reste pratiquement encore empirique.

On sait que les substances dites galactogènes ont une efficacité très variable ; l'influence du régime est également inconstante et seuls les moyens mécaniques destinés à exciter la sécrétion glandulaire fournissent assez souvent des résultats satisfaisants. C'est ainsi que la succion régulière du mamelon suiviè de l'expression totale des seins à intervalles fixes déterminent progressivement une excitation glandulaire croissante, et il est assez fréquent de voir de cette façon la quantité de lait augmenter dans des proportions notables.

Mais il s'agit là de moyens lents, souvent pénibles, auxquels nombre de femmes refusent de recourir assez régulièrement pour obtenir le résultat souhaité.

Par contre, la technique d'irradiations mammaires, préconisée pour la première fois par STOLTE et WIENER en 1928, est d'applieation simple, et les premiers résultats obtenus par les auteurs allemands paraissaient encourageants.

La technique que nous avons employée est sensiblement analogue à celle qui avait donné à STOLTE et WIENER, puis à VoGT, des succès presque eonstants.

Nous avons pratiqué des séances quotidiennes d'irradiation mammaire pendant une durée progressivement croissante de 5 à 30 ou 35 minutes selon la tolérance individuelle, la lampe restant écartée de 80 , puis $70 \mathrm{~cm}$. de la surface des seins vers lesquels les rayons étaient dirigés cependant que le reste du corps était protégé. Une légère couche de vaseline isolait le mamelon. Les réactions locales cutanées, variables selon les sujets, ont pu être assez intenses parfois pour empêcher d'augmenter la durée d'irradiation au delà de certaines limites (érythème, prurit ou pigmentation). Parmi les nombreuses femmes que nous avons ainsi traitées, nous relaterons seulement les observations 
de celles qui ont été suivies assez longtemps et régulièrement pour qu'il soit possible d'apprécier l'efficacité de la thérapeutique.

Sur 15 cas bien suivis, nous obtenons 10 succès et 5 échecs. Notons de suite que ces succès eux-mêmes ne paraissent pas aussi éclatants que ceux qu'avaient enregistrés les auteurs de la méthode. Jamais nous n'avons observé de montées laiteuses brusques et massives, distendant les seins après l'application des R. U. V., même alors qu'il s'agissait de bonnes nourrices ehez qui nous avions institué le traitement dans un but expérimental; dans tous les cas, la progression fut lente.

\section{1. - Augmentation de la quantité de lait sous l'influence des $R . U$. V.}

Chez 10 femmes le résultat fut positif :

10 $M^{\text {me }} H$., après avoir nourri 3 jours son enfant, a présenté une lymphangite du sein gauche qui a nécessité l'arrêt de la lactation de ce côté. Depuis un mois elle ne donne que le sein droit.

Après la $1^{\text {re }}$ séance de rayons (5 minutes) l'expression du sein gauche fournit $10 \mathrm{gr}$.

Après la $3^{\mathrm{e}}$ séance les montées laiteuses sont suffisantes pour permettre la suppression des biberons de complément de $50 \mathrm{gr}$, donnés jusqu'alors.

Au cours des séances ultérieures (jusqu'à 20 minutes) on ne note plus d'augmentation nette : les montées laiteuses demeurent irrégulières, mais atteignent à certaines têtées $50 \mathrm{gr}$.

$2^{\circ} M^{\text {me }} C$. suit régulièrement le traitement jusqu'à une durée d'irradiation de 30 minutes.

Les tétées augmentent progressivement de 10 à 60 gr. (ehiffre obtenu après 20 minutes d'irradiation), puis demeurent stationnaires. Après cessation du traitement la quantité de lait ne subit plus de variations notables.

$3^{\circ} M^{\text {me }} T$. (enfant âgé de 15 jours).

Séances de R. U. V. de 5 à 25 minutes.

Montées laiteuses irrégulières :

Au début $5 \mathrm{gr}$.

Augmente jusqu'à $30 \mathrm{gr}$. après quelques oscillations.

Après 13 séances allaitement mixte possible :

Tétées abondantes le matin ( 80 à $100 \mathrm{gr}$.)

Tétées complétées le soir (30 à 40 gr.).

$4^{\circ} M^{\text {me }} R$. (enfant âgé de 1 mois.)

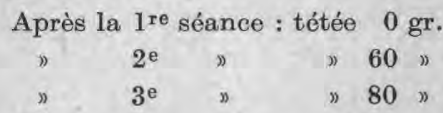

Irradiations interrompues pour érythème accentué. Allaitement exclusif au sein demeure possible pendant un mois, puis allaitement mixte pour raisons sociales.

5o $M^{\text {me }} L$. (enfant âgé de 2 mois).

Mamelon ombiliqué à gauche. 
$10^{\mathrm{e}}$ grossesse. Le $1^{\text {er }}$ et le $3^{\mathrm{e}}$ enfant ont pu prendre le sein gauche à l'aide d'un tire-lait pendant 3 mois. Pour le $2^{\mathrm{e}}$ et le $4^{\mathrm{e}}$ enfant, pas de montée laiteuse de ce côté. Les 3 derniers ont été sevrés à 2 mois, la quantité de lait restant très insuffisante ( $20 \mathrm{gr}$. environ).

Le dernier enfant prend $40 \mathrm{gr}$. au sein droit et ne tette pas le sein gauehe.

Séances de R. U. V. régulières, mais durée d'irradiation lentement augmentée à cause de réactions locales :

$\begin{array}{cc}\begin{array}{c}\text { Tétées au sein gauche } \\ \text { (moyenne) }\end{array} & \begin{array}{c}\text { Durées des } \\ \text { séances }\end{array} \\ 5 & 5 \\ 70 & 10^{\prime} \\ 100 & 15 \\ 80 & 15 \\ 50 & 15\end{array}$

(la mère ne complète plus que 2 tétées par jour)

(croissance pondérale quotidienne de 1 enfant : 31 gr.)

60

90

Allaitement exclusif aux 2 seins se poursuit normalement après l'arrêt des rayons.

$6^{\circ} M^{\text {me }} B$. (enfant âgé de 2 mois).

Séances de R. U. V. de 5 à 30 minutes ( 4 fois).

$\mathrm{Au}$ début tétées de $10 \mathrm{gr}$. environ.

Augmentation progressive jusqu'à 60 gr., puis quantité demeure à peu près stationnaire avec de légères oscillations.

Les 3 dernières observations concernent des femmes ayant une quantité de lait suffisante mais que l'on s'efforçait d'augmenter pour leur permettre de fournir du lait à d'autres enfants que le leur.

$7^{\circ} M^{\text {me }} L$.

Irradiations 5 à 30 minutes.

Avant le traitement une tétée pesée plusieurs jours de suite : 40 à $60 \mathrm{gr}$.

A la même heure augmentation progressive après le début du traitement : $90,80,80,117,140,120$.

Notons que la tétée immédiatement consécutive à la séance d'irradiation était toujours plus faible ( 40 à $50 \mathrm{gr}$.), mais les tétées ultérieures redevenaient abondantes.

$8^{\circ} M^{\text {me }} G$.

Irradiations 5 à 20 minutes.

Avant le traitement : tétées de $100 \mathrm{à} 120 \mathrm{gr}$.

Pendant le traitement : 110, 110, 130, 160, 200.

(Même phénomène que dans l'observation 7.)

$9^{\circ} M^{\text {me }} S$. hospitalisée à Trousseau. Allaite son enfant âgé de 1 mois $1 / 2$. Avant le traitement :

Têtées du matin 70 à $80 \mathrm{gr}$. complétées. 
A midi 90 à $100 \mathrm{gr}$.

Le soir 60 à 80 gr. complétées.

Irradiations du 12 au 17 juin de 5 ' à $30^{\circ}$.

Augmentation progressive des quantités de lait.

A partir du 15 juin les tétées du matin sont de $110 \mathrm{gr}$.

A midi 100 à $130 \mathrm{gr}$.

Le soir 90 à $100 \mathrm{gr}$.

Cette augmentation apparaît d'abord irrégulièrement, puis se régularise :

\begin{tabular}{|c|c|c|c|c|c|}
\hline 15 juin & matin & 110 & à midi & 100 & le soir \\
\hline $16 "$ & n & 100 & $n$ & 130 & $n$ \\
\hline $17 》$ & » & 100 & " & 120 & x \\
\hline $19 "$ & ) & 100 & $n$ & 120 & j) \\
\hline
\end{tabular}

L'alimentation de l'enfant est poursuivie ultérieurement au sein non complété, avec une croissance pondérale normale. Le contrôle de la quantité de lait fourni par la mère quelques jours après la fin du traitement montre une persistance des chiffres élevés observés (100 à $130 \mathrm{gr}$.).

$10^{\circ} M^{\text {me }} L e b$. (enfant âgé de 6 semaines).

12 séances de R. U. V. de 5 à $35^{\text {' }}$.

Augmentation progressive de la quantité totale de lait fourni en 24 heures.

Avant le traitement quantité de lait par $24 \mathrm{~h}$., environ $450 \mathrm{gr}$.

Irrad. $\quad 5^{\prime} \quad 1^{\prime} 0^{\prime} \quad 15^{\prime} \quad 20^{\prime} \quad 20^{\prime} \quad 20^{\prime} \quad 25^{\prime} \quad 25^{\prime}, 30^{\prime} \quad 35^{\prime} \quad 35^{\prime} \quad 35^{\prime}$

Lait on 24 h. $450-575-490-520-490-535-570-595-605-580-610-600$

Dans 5 cas le traitement. fournit des résultats très incomplets ou nuls.

Mme L. - Moyenne des têtées avant le traitement : $10 \mathrm{gr}$.

Pendant les trradiations quelques montées laiteuses irrégulières.

A la fin du traitement aucune amélioration : sevrage.

$M^{\text {me }} S$. - Pas de montée laiteuse supérieure à celles observées antérieurement. Interruption après 5 séances.

Trois autres femmes, dont la sécrétion lactée était très insuffisante, sont soumises aux R. U. V. pendant 8 à 10 jours sans augmentation nette de leur quantité de lait.

Pour certaines de ces femmes, le traitement institué normalement dut être interrompu après la $5^{\mathrm{e}}$ et la $8^{\mathrm{e}}$ séance, par suite du découragement des jeunes mères. D'autres échees ont été observés chez des nourrices venues très irrégulièrement aux rayons.

Mais, dans 3 cas, le traitement effectué de façon suffisamment régulière et prolongée aboutit cependant à un échec.

Ainsi, du point de vue quantitatif, un traitement par irradiations bien conduit, avec séances quotidiennes et progressives non interrompues et assez prolongées, paraît le plus souvent, quoique non constamment, exciter une sécrétion lactée antérieurement insuffsante. On peut même espérer un résultat favorable chez certaines femmes qui ont présenté une agalactie ou une hypogalactie notable lors 
d'allaitements antérieurs (obs. 5), ou chez des nourrices ayant interrompu l'allaitement depuis quelque temps (obs. I et 5).

Parfois cependant, sans cause décelable, le résultat du traitement se montre insuffisant ou nul.

Sans pouvoir par conséquent affirmer le succès constant de cette thérapeutique, ses résultats sont cependant assez encourageants pour mériter qu'on l'entreprenne.

Signalons d'ailleurs que, dans quelques cas, nous avons obtenu une augmentation de la sécrétion lactée au cours d'irradiations générales, et, ainsi que nous le verrons, l'influence des R. U.V. sur l'état général de la nourrice n'est pas négligeable. Chez une femme en particulier qui désirait sevrer son enfant par suite d'un état de fatigue accentué, les séances de R. U. V. sur toute la surface du corps, pratiquées à titre de thérapeutique reconstituante, amenaient chaque fois une réplétion des seins, alors que les jours intermédiaires aux irradiations, aucune montée laiteuse ne se produisait plus.

2. - Etude des modifications qualitatives du lait sous l'influence des R.U.V.

Chez un certain nombre des nourrices en observation, du lait a été prélevé tous les jours avant et après les séances d'irradiations, afin de rechercher les variations qualitatives survenues scus l'influence des rayons.

Les dosages pratiqués par M. ZizINE et ses coilaboratrices ont porté sur l'extrait sec, les cendres, le beurre, le lactose et la caséine.

Dars l'ensemble, les variations ont été très faibles, et la 1ichesse du lait en matières minérales ou organiques n'a nullement paru augmenter.

La quantité totale de substances nutritives sécrétées s'est certes accrue par suite des variations quantitatives générales, mais nulle concentration n'a été observée.

Notons cependant que l'étude de la tétée consécutive aux applications de R. U.V., tétée le plus souvent quantitativement diminuée, révélait une augmentation de la concentration.

Ainsi dans l'ensemble la sécrétion nutritive demeurait augmentée même lorsque la quantité de liquide subissait des variations régressives passagères.

Par exemple :

$M^{\text {me }}$ G. (obs. 8). R. U. V. faits immédiatement après la tétée de 11 heures

\begin{tabular}{|c|c|c|c|c|c|}
\hline L & tétée de & $\begin{array}{l}11 \text { h. } 120 \mathrm{gr} \text {. } \\
14 \text { h. } 60 \text { " }\end{array}$ & $\begin{array}{c}\text { Extrait sec } \\
\qquad \begin{array}{c}141 \\
161\end{array}\end{array}$ & $\begin{array}{c}\text { Beurre } \\
54 \\
67\end{array}$ & $\begin{array}{c}\text { Lactose } \\
\quad 64 \\
70\end{array}$ \\
\hline Le $4 / 1$ & $"$ & 11 h. 110 n & 148 & 60 & 65 \\
\hline & $"$ & $14 \mathrm{~h} .55$ & 162 & 68 & 70 \\
\hline $7 / 1$ & $n$ & $11 \mathrm{~h} \cdot 160 \mathrm{n}$ & 147,7 & 52 & 66 \\
\hline & " & 14 h. 60 ») & 151 & 56 & 71 \\
\hline
\end{tabular}


La concentration des diverses substances restant sensiblement analogue, leur sécrétion est bien réellement augmentée parallelement à l'augmentation de la quantité totale de lait.

Ainsi Mme L. (obs. 7).

Tétées $\quad \begin{gathered}\text { Extrait sec } \\ \text { pour } 1000\end{gathered}$

Au début :
135

137

133

135
Cendres
pour 1000

$\begin{array}{ccc}\text { Beurre } & \text { Lactose } & \text { Caséine } \\ \text { pour } 1009 & \text { pour I000 } & \text { pour } 1000\end{array}$

pour 1009 pour 1000

64

33

2,44n $\quad 35 \quad 64 \quad 33$

Pendant le traitement :

$\begin{array}{rlllll}80 & 137 & 2,26 & 34 & 72 & 28 \\ 117 & 133 & 2,54 & 34 & 69 & 28 \\ 140 & 135 & 2,47 & 35 & 64 & 34\end{array}$

A la fin du traitement :

120

142

2,17

42

66

31

3. - Valeur antirachitique du lait aprés irradiation mammaire.

Le lait de femme a habituellement une valeur antirachitique presque nulle.

Hess et WeInstock ont constaté que le rat n'est pas protégé par 20 gouttes de lait ajoutées quotidiennement à un régime rachitigène

Le beurre préparé avec du lait de femme, ajouté à la ration d'un rat dans la proportion de $5 \%$, n'évite pas l'apparition des lésions rachitiques, ainsi que l'ont montré Lesné et Vaguiano. Mais Hess et Whinstock, Sherman, Gerstenberger, avaient noté la modifieation du lait par les irradiations des nourrices, et HoRT et STEENBOCK constataient l'apparition du facteur antirachitique dans le lait des vaches après exposition de ces animaux sous la lampe de Quartz.

Nous avons recherché cette modification qualitative du lait $\mathrm{chez}$ quelques-unes des femmes traitées par irradiation locale mammaire.

Les laits de Mme Leb (obs. 10) et de Mme B. (obs.6.) ont été étudiés à ce point de vue comparativement à du lait d'une nourrice non irradiée.

3 groupes de 4 rats ont été soumis à un régime rachitigène auquel on ajoutait quotidiennement 2-4-6-8 gouttes de lait à étudier.

Après 15 jours, les animaux sont sacrifiés et on examine leurs thorax.

Dans le $1^{\text {er }}$ groupe qui a reçu du lait de nourrice non irradiée, on observe un chapelet de nodosités costales volumineúses avec déformation thoracique nette, aussi bien chez le rat ayant absorbé 8 gouttes de lait par jour que chez celui qui n'en ingérait que 2.

Par contre les 2 autres groupes de rats mis parallèlement au même régime ont été protégés par le lait de nourrice irradiée. Il exište de très minimes nodosités costales sans déformation thoracique chez les 8 animaux en expérience ; la protection a donc été réalisée dans une large mesure même avec des doses très minimes ( 2 gouttes). 
La valeur anti-rachitique du lait des femmes soumises à l'irradiation mammaire est donc comparable, sinon supérieure à celle développée par l'irradiation générale.

Le mécanisme de cette action des rayons est difficile à élucider. Nous avons vérifié l'absence d'activité semblable d'une source calorifique banale (air chaud).

Par contre l'irradiation générale paraît exercer une action du même ordre quoique plus faible.

L'influence des rayons U. V. sur la nutrition générale favorise vraisemblablement le fonctionnement glandulaire et celui de la glande mammaire en particulier.

En outre une action exeitante plus spéciale paraît être déterminée par l'irradiation directe des téguments recouvrant la glande mammaire

La limitation de l'irradiation permettant de la régler et de la surveiller de plus près facilite l'application de doses plus rapidement accrues.

Le rôle de la congestion mammaire par action calorifique locale est peut-être à considérer aussi à titre accessoire, et expliquerait dans une certaine mesure l'inhibition passagère et relative observée chez certaines nourrices lors de la tétée consécutive à l'application des R. U. V.

En conclusion l'irradiation mammaire, facile à réaliser paraît une méthode pratique et souvent efficace d'excitation d'une sécrétion lactée insuffisante, à condition toutefois de poursuivre le traitement de façon régulière et d'employer des doses suffisantes.

Le lait fourni par ces nourrices n'est pas modifié dans sa composition chimique mais subit une variation biologique qualitative caractérisée par l'apparition du facteur antirachitique en quantités notables.

En outre l'influence eutrophique de l'irradiation s'exerce sur l'état général de la nourrice malgré la limitation des irradiations. Le mode d'activité des irradiations mammaires reste encore imprécis et il est impossible actuellement de spécifier en quoi cette action locale diffère de l'influence exercée par les irradiations générales, et même d'affirmer qu'il existe une différence essentielle entre les 2 processus.

Mais dans l'ensemble cette méthode favorisant la sécrétion lactée peut être utilisée sans nul risque et présente le double avantage d'exercer une action favorable sur la quatité et la quanlité du lait fourni à l'enfant, tout en améliorant l'état général de la mère. 